\title{
Rene, ADPKD e Covid-19: il doppio ruolo della fragilità renale
}

\author{
Maria Teresa Sciarrone Alibrandi, Marta Vespa \\ Divisione di Nefrologia Dialisi e Ipertensione, IRCCS Ospedale san Raffaele, Milano - Italia
}

\begin{abstract}
Kidney, ADPKD and Covid-19: the double role of renal fragility
The Corona Virus Disease 19 (Covid-19) is an infectious disease caused by SARS-CoV-2 (Severe Acute Respiratory Syndrome Coronavirus 2). By now, the pandemic has already led 318000 people to their death and our knowledge of this pathogen is still in its infancy. What we know so far is that older patients suffering from different comorbidities, such as heart failure, COPD and tumors, are more likely to develop severe complications from which they might perish. But the role of chronic kidney disease is still not clear. What we know is that CKD patients are more susceptible to infections, both viral and bacterial, compared to the rest of population. It has been recently highlighted that the immune response might be the real cause of damage in the lung tissues and not just the virus itself, therefore, the better the immune response, the greater the damage. Quite paradoxically, being chronically renal impaired and suffering from a delayed T-Cell response might actually be beneficial, avoiding a massive inflammatory reaction followed by SARS. According to a recent survey led by the SIN (Italian Society of Nephrology), only $2.8 \%$ of Covid patients is under hemodialysis treatment. Unfortunately, we do not have more detailed data on every single specific form of CKD. For instance, there is no a clear relation between Covid-19 an ADPKD. What might be inferred is the following: Covid uses the ACE2 receptors on cell membranes to "lock on" its target. It is a well-established fact that the RAAS is more active in ADPKD patients, but if it is a matter of hyper-activation or hyper-expression of ACE2 receptors is not clear. Also, the fact that these patients are usually on drug treatment with an ACE inhibitor or an ARB might have some implications that must be analyzed.
\end{abstract}

Keywords: ADPKD, Covid-19, Kidney

La diffusione dell'infezione da nuovo coronavirus (definito dall'OMS "SARS-CoV-2") ha recentemente messo in allarme la popolazione generale, cambiandone radicalmente le abitudini.

Le conoscenze su questa nuova infezione umana, comparsa solo a dicembre 2019 sulla scena mondiale, sono ancora premature e in continua evoluzione; la sindrome clinica (cioè I'insieme delle manifestazioni dell'infezione da SARS-CoV-2) è stata nominata Covid-19.

Occorre ancora molta cautela nell'offrire suggerimenti che non possano basarsi su evidenze e osservazioni specifiche, solide e rigorose (1).

Received: May 25, 2020

Accepted: June 12, 2020

Published online: July 06, 2020

Indirizzo per la corrispondenza:

Maria Teresa Sciarrone Alibrandi

Divisione di Nefrologia Dialisi e Ipertensione

IRCCS Ospedale san Raffaele

Via Olgettina 60

20132 Milano - Italia

sciarronealibrandi.mariateresa@hsr.it
La trasmissione da persona a persona del SARS-CoV-2 è stata dimostrata e sembra verificarsi soprattutto tramite goccioline, anche da soggetti asintomatici. Un parametro importante in un'epidemia infettiva è il cosiddetto R0, ovvero il "numero di riproduzione di base", che rappresenta il numero medio di infezioni secondarie prodotte da ciascun individuo infetto in una popolazione completamente suscettibile, cioè mai venuta a contatto con il virus. Questo parametro misura la potenziale trasmissibilità di una malattia infettiva. In altre parole, se il cosiddetto RO di una malattia infettiva è pari a circa 2, significa che, in media, un singolo malato infetterà due persone. Quanto maggiore è il valore di RO tanto più elevato è il rischio di diffusione dell'epidemia. Da quanto emerso per Covid-19, queste stime sono comprese tra 1,4 e 3,8 nelle aree colpite, con una media pari a 2 circa, indicativa, quindi, di contagiosità medio-elevata $(1,2)$.

Per quanto riguarda le manifestazioni cliniche della malattia, esistono diverse modalità di presentazione e l'andamento è oltremodo variabile.

La maggior parte delle infezioni da Sars-CoV-2 non ha caratteristiche severe, ma è stato stimato che circa 1 paziente su 5 può presentare una forma grave di malattia, caratterizzata da polmonite con insufficienza respiratoria, che richiede 
ventilazione assistita fino all'intubazione e spesso associata a complicanze di natura tromboembolica (3).

Il tasso di mortalità riportato varia tra il $6 \%$ e $<1 \%$. Al momento, i fattori aggravanti noti sono l'età avanzata ( $>65$ anni) e la presenza di altre patologie croniche concomitanti, come le malattie cardiovascolari e respiratorie e i tumori. Sono stati comunque osservati casi di pazienti che hanno manifestato forme severe di malattia pur in assenza dei fattori di rischio succitati (1-5).

A tutt'oggi, non è noto se l'insufficienza renale cronica abbia un peso in quella che è la modalità di presentazione di Covid-19. È risaputo, infatti, che i pazienti affetti da insufficienza renale cronica sono più a rischio di sviluppare infezioni in generale. I pazienti dializzati più nello specifico presentano un sistema immunitario compromesso e questo li rende particolarmente suscettibili a sviluppare infezioni di origine sia batterica che virale in modo più severo rispetto alla popolazione generale (4-6).

Alcuni studi recenti suggeriscono, però, che sia proprio la risposta immunitaria a giocare un ruolo cruciale nella patogenesi delle forme più gravi di Covid-19, in particolar modo la risposta immunitaria mediata dai linfociti T. II fatto che l'uremia sia associata a una ridotta risposta immunitaria di tipo sia linfocitario che granulocitario potrebbe, quindi, paradossalmente, scongiurare l'insorgenza di forme più gravi di malattia proprio in un gruppo di pazienti notoriamente più fragili (6).

Esistono segnalazioni di autori cinesi che riportano una minore incidenza di forme severe di malattia tra i pazienti sottoposti a trattamento dialitico (7).

Secondo una survey effettuata di recente dalla Società Italiana di Nefrologia, la percentuale di pazienti con Covid-19 in emodialisi è del $2,8 \%$ e quella dei pazienti in dialisi peritoneale è del $2,4 \%$.

I pazienti dializzati in caso di infezione da Covid-19 presentano, in genere, linfopenia di grado minore (riduzione del numero dei linfociti nel sangue), livelli di citochine infiammatorie più bassi e, in genere, una forma meno aggressiva di malattia. Questo riscontro avvallerebbe, quindi, l'ipotesi che i pazienti dializzati probabilmente non sono in grado di sviluppare una reazione infiammatoria cosi importante, come osservato nei soggetti con funzione renale normale, e questo determinerebbe proprio un'espressione più moderata di Covid-19 (7).

Per quanto riguarda, invece, gruppi selezionati di pazienti e stratificati per patologia renale, non ci sono dati specifici.

In caso di malattia del rene policistico, per esempio, allo stato attuale delle conoscenze non ci sono segnalazioni che pazienti affetti da ADPKD in presenza di funzione renale normale siano più a rischio di sviluppare forme severe di Covid19 rispetto alla popolazione generale. Nei pazienti affetti da ADPKD, infatti, non si sa se possano esistere fattori predisponenti o, viceversa, magari fattori addirittura protettivi verso la malattia, come qualche osservazione sul modello animale potrebbe suggerire.
È noto, infatti, che SARS-CoV-2 utilizza il recettore ACE2 per infettare le cellule, sito di legame per ACE2, uno degli enzimi centrali nel sistema renina-angiotensina-aldosterone (RAAS), che regola la pressione arteriosa, i volumi corporei, l'equilibrio elettrolitico e le resistenze vascolari sistemiche $(8,9)$.

Nei polmoni, l'attivazione del RAAS locale può influenzare la patogenesi del danno polmonare attraverso meccanismi multipli, come un aumento della permeabilità vascolare e alterazioni delle cellule epiteliali alveolari. Questo effetto, quindi, mediato localmente dal virus, ha probabilmente un ruolo patogenetico nel danno polmonare. È noto che i pazienti affetti da ADPKD hanno di per sé un'iperattivazione del RAAS, mentre non è stata studiata la presenza di una maggiore o minore espressione del recettore ACE2, anche mediata da farmaci ACE inibitori o sartani, assunti cronicamente, nella stragrande maggioranza dei casi, a scopo antiipertensivo o nefroprotettore.

È possibile un collegamento tra queste osservazioni? L'espressione del recettore ACE2 nelle cellule bersaglio del virus è aumentata dall'uso di ACE inibitore/bloccante del recettore dell'angiotensina e, quindi, il paziente è più a rischio per un decorso grave o, viceversa, questa terapia cronica può rappresentare un fattore protettivo (10)?

Sarebbe, quindi, interessante valutare, nell'ambito di questa popolazione selezionata, l'impatto dell'infezione Covid-19 e la sua severità di manifestazioni e di complicanze rispetto alla popolazione generale o a pazienti affetti da altre nefropatie.

Inoltre, per quanto riguarda il coinvolgimento renale diretto in corso di Covid-19, è importante sottolineare come le forme più severe della malattia possano determinare una sofferenza a questo livello anche in pazienti non nefropatici o con una funzione renale solo lievemente compromessa.

I meccanismi attraverso i quali questo processo potrebbe avvenire sono almeno due. In maniera diretta con un meccanismo di tossicità mediato dal virus. Alcuni studi autoptici hanno, infatti, dimostrato un danno renale acuto, evidenziando la presenza di particelle di coronavirus nei reni che sono particolarmente ricchi di recettori ACE2 (fino a 100 volte di più di quanto riscontrato nel tessuto polmonare), che rappresentano, come detto sopra, la porta d'ingresso del virus nelle cellule.

Esiste, probabilmente, anche un meccanismo legato alla grave reazione infiammatoria sistemica descritta sopra. Citochine e mediatori dell'infiammazione possono, infatti, danneggiare il parenchima renale sia direttamente sia indirettamente, anche con induzione di fibrosi.

Anche in caso di coinvolgimento renale secondario all'infezione da SARS-CoV-2 sarebbe interessante valutarne l'andamento in relazione anche alla nefropatia di base. Come accennato in precedenza, una differente espressione di recettori ACE2 a questo livello potrebbe implicare un differente decorso della malattia. 
In conclusione, quindi, si può affermare che, dati l'andamento spesso non prevedibile, l'elevata diffusione della malattia e il suo tasso di mortalità relativamente elevato, è sicuramente importantissimo comprendere i fattori predisponenti per lo sviluppo delle forme più gravi.

La maggior parte dei dati che riguardano le manifestazioni cliniche, il trattamento e la prognosi dei pazienti deriva dall'osservazione di piccoli gruppi di pazienti spesso non selezionati, e non è ancora disponibile un'osservazione su larga scala, dato l'esordio relativamente recente di questa patologia (9).

Dunque, l'obiettivo principale degli studi è caratterizzare a tutto tondo i pazienti affetti da Covid-19, per evidenziare quali fattori clinici pregressi, genetici o ambientali possano predisporre allo sviluppo di forme più severe di malattia o, viceversa, possano proteggere dalle forme gravi e, quindi, predisporre a un andamento più lieve o, addirittura, asintomatico.

Per quanto riguarda l'approccio terapeutico, non esiste ancora una cura mirata per la malattia, ma iniziano a essere disponibili dati sui farmaci utilizzati e potenzialmente utilizzabili $(11,12)$.

Idealmente, i pazienti andrebbero trattati con terapie antivirali efficaci. Malgrado ciò, le molecole al momento utilizzate non sono state sviluppate per trattare le infezioni da coronavirus e l'esperienza clinica è scarsa $(11,12)$.

La comprensione della variabilità di manifestazioni e di evoluzione della malattia tra soggetto e soggetto è, pertanto, alla base della ricerca di una terapia efficace e risolutiva.

Non ultimo, in ambito nefrologico è bene ricordare l'importanza di stratificare i pazienti in relazione alla nefropatia di base, al fine di individuare eventuali ulteriori fattori di rischio o protettivi nelle manifestazioni di Covid-19, considerato il ruolo del RAAS, sistema ormonale cruciale sia per il rene che per questa emergente patologia.

\section{Disclosures}

Conflict of interest: The authors declare no conflict of interest. Financial support: This research received no specific grant from any funding agency in the public, commercial, or not-for-profit sectors.

\section{Bibliografia}

1. Zhou F, Yu T, Du R et al. Clinical course and risk factors for mortality of adult inpatients with COVID-19 in Wuhan, China: a retrospective cohort study. The Lancet 2020;395:1054-62.

2. $\mathrm{Xu} \mathrm{Z}$, Shi L, Wang $\mathrm{Y}$ et al. Pathological Findings of COVID-19 Associated With Acute Respiratory Distress Syndrome. The Lancet Respir Med. 2020;8:420-22.

3. Ruan $Q$, Yang $K$, Wang W, Jiang $L$, Song J. Clinical predictors of mortality due to COVID-19 based on an analysis of data of 150 patients from Wuhan, China. Intensive Care Med. 2020;46: 846-48.

4. Wang D, Hu B, Hu C. Clinical Characteristics of 138 Hospitalized Patients With 2019 Novel Coronavirus-Infected Pneumonia in Wuhan, China. JAMA;323:1061-69.

5. Bouadma L, Lescure F-X, Lucet J-C et al. Severe SARS-CoV-2 Infections: Practical Considerations and Management Strategy for Intensivists. Intensive Care Med. 2020;46:579-82.

6. Xianghong $Y$, et al. Diagnosis and Treatment of COVID-19: Acute Kidney Injury Cannot Be Ignored. Natl Med J China. 2020;100:1205-8.

7. Rui W, Liao C, He H et al. COVID-19 in Hemodialysis Patients: A Report of 5 Cases. Am J Kidney Dis. 2020;76:141-3.

8. Guan WJ, Ni ZY, Hu Y et al. Clinical Characteristics of Coronavirus Disease 2019 in China. N Engl J Med. 2020;382:1708-20.

9. Zhang $Y$ et al. Vital surveillances: the epidemiological characteristics of an Outbreak of 2019 Novel Coronavirus Diseases (Covid-19) - China CDC Weekly 2020;2:113-122.

10. Sommerstein $R$, Kochen $M$, Messerli FH, Grani $C$ et al. Coronavirus Disease 2019 (COVID-19): Do AngiotensinConverting Enzyme Inhibitors/Angiotensin Receptor Blockers Have a Biphasic Effect? JAHA. 2020 Apr. doi.org/10.1161/JAHA. 120.016509.

11. Huang C, Wang Y, Li X et al. Clinical features of patients infected with 2019 novel coronavirus in Wuhan, China. Lancet. 2020; 395:497-506.

12. Stebbing J, Phelan A, Griffin I et al. COVID-19: combining antiviral and anti-inflammatory treatments. The Lance Infectious Diseases 2020;20:400-402. 